\title{
(596) Scheila in outburst: a probable collision event in the Main Asteroid Belt
}

F. Moreno

Instituto de Astrofísica de Andalucía, CSIC, Glorieta de la Astronomía s/n, 18008

Granada, Spain

fernando@iaa.es

J. Licandro

Instituto de Astrofísica de Canarias, c/Vía Láctea s/n, 38200 La Laguna, Tenerife, Spain, and Departamento de Astrofísica, Universidad de La Laguna, E-38205 La Laguna,

Tenerife, Spain

J.L. Ortiz

Instituto de Astrofísica de Andalucía, CSIC, Glorieta de la Astronomía s/n, 18008

Granada, Spain

L.M. Lara

Instituto de Astrofísica de Andalucía, CSIC, Glorieta de la Astronomía s/n, 18008

Granada, Spain

V. Alí-Lagoa

Instituto de Astrofísica de Canarias, c/Vía Láctea s/n, 38200 La Laguna, Tenerife, Spain, and Departamento de Astrofísica, Universidad de La Laguna, E-38205 La Laguna,

Tenerife, Spain

O. Vaduvescu

Isaac Newton Group of Telescopes, Apdo. de Correos 321, E-38700 Santa Cruz de la Palma, Canary Islands Spain, and Instituto de Astrofísica de Canarias, c/Vía Láctea s/n, 38200 La Laguna, Tenerife, Spain

N. Morales

Instituto de Astrofísica de Andalucía, CSIC, Glorieta de la Astronomía s/n, 18008

Granada, Spain

A. Molina

Instituto de Astrofísica de Andalucía, CSIC, Glorieta de la Astronomía s/n, 18008

Granada, Spain, and Departamento de Física Aplicada, Universidad de Granada, Fuentenueva s/n, 18071 Granada, Spain 
and

Lin, Z.-Y.

Instituto de Astrofísica de Andalucía, CSIC, Glorieta de la Astronomía s/n, 18008

Granada, Spain

\begin{abstract}
Images of asteroid (596) Scheila have been acquired at various dates after the discovery of the 2010 outburst. Assuming a short-duration event scenario, as suggested by the quick vanishing of the dust tail brightness with time, and integrating numerically the equation of motion of individual particles ejected from the surface, we have developed a tail model from which we estimate the parameters associated to the geometry of the ejection, the size distribution, and the velocity distribution of the ejected particles, as well as the total mass ejected. We found a weak inverse power-law dependence of ejection velocity versus particle radius, with velocities ranging from 50 to $80 \mathrm{~m} \mathrm{~s}^{-1}$ for particle radii in the range $5 \mathrm{~cm}$ to $8 \times 10^{-5} \mathrm{~cm}$, respectively. These velocities are very different from those expected from ice sublimation at the asteroid heliocentric distance ( $\sim 3 \mathrm{AU})$, and suggest a collision scenario as a likely cause of the outburst. We found that the ejected particles are distributed in size following a power law of index -3 , and, based on the ejecta mass and scaling laws, the impactor size is estimated at 30-90 $\mathrm{m}$ in radius, assuming an impact velocity of $\sim 5 \mathrm{~km} \mathrm{~s}^{-1}$, and the same density $\left(1500 \mathrm{~kg} \mathrm{~m}^{-3}\right)$ for the asteroid as for the projectile. We have inferred an asymmetry in the ejecta along the axis normal to the asteroid orbit plane, a likely indicator of an oblique impact. The impact is estimated to have occurred on November 27th, with an accuracy not better than \pm 3 days.
\end{abstract}

Subject headings: minor planets, asteroids: general — asteroids: general — asteroids: individual(596 Scheila) — methods: data analysis

\title{
1. Introduction
}

(596) Scheila is a large Main-Belt Asteroid (113 $\mathrm{km}$ in diameter) with very low $\left(p_{v}=0.038\right.$, Tedesco et al. 2002) geometric albedo. It has been classified as a D-type asteroid based on its visible and near-ir spectroscopy (Licandro et al. 2011). On December 11.4 2010, the asteroid 
displayed a surprising comet-like appearance, constituting the seventh of the so-called MainBelt Comets (Hsieh \& Jewitt 2006). The outburst was discovered by Larson (2010) from Catalina Sky Survey (CSS), who reported the object as having a total magnitude $V=13.4$, By inspection of the CSS archive images, it was determined that the outburst must have occurred sometime between November 11th and December 3rd, 2010 (Larson 2010). Right after the outburst detection many amateur and professional astronomers performed observations of the object, which displayed a well-defined bifid tail in the anti-sunward direction, consisting, in principle, of dust grains being blown away by radiation pressure. The tail was clearly seen since detection, but the brightness tend to vanish quickly after a few days, becoming undetectable after some three weeks after discovery. This scenario could be compatible with a short-duration event, such as a collision with another body, but other possibilities, such as a "regular", short-term, cometary-like outburst, or a sudden mass loss from rotational instability cannot be ruled out in principle. From analysis of HST images, Jewitt et al. (2011) conclude that either rotational instability or electrostatic ejection of regolith cannot be responsible of the observed mass loss, which is most simply explained by a collision with a previously unknown asteroid of $\sim 35 \mathrm{~m}$ in diameter. Bodewits et al. (2011) analyzed a Scheila spectrum between 290 and $500 \mathrm{~nm}$, and found no gaseous emissions, coming also to the conclusion that Scheila was impacted by another main belt asteroid less than $100 \mathrm{~m}$ in diameter.

In this paper we report images of the asteroid at different dates, and make an interpretation on the basis of a short-term event using a forward Monte Carlo dust tail code, in order to provide estimates of (i) timing, and geographic location of the event on the asteroid surface, (ii) particle ejection velocity law, (iii) particle size distribution, and (iv) total ejected mass.

\section{Observations and data reduction}

Images of (596) Scheila were obtained since December 13th, 2010 till January 25th, 2011, using telescopes at various observatories. In addition to professional images, and for completeness, we have added to our image set an image taken by J. Brimacombe at the New Mexico Skies Observatory (NMSO). Table I shows the log of the observations, along with some relevant parameters. Reduction was performed using standard techniques, including bias subtraction, flat fielding, and calibration using zero-points derived from field stars and the USNO-B1.0 catalog (Monet et al. 2003), which provides a photometric accuracy of $\sim 0.3$ mag. In all cases, red Johnson filters were used, in order to minimize possible contamination by emission lines in the blue and UV regions. The asteroid spectrum taken on December 
15th by Bodewits et al. (2011) shows no evidence for the presence of gaseous emissions, so that all the brightness we see in the tails are due to solar light singly scattered by dust.

The available images each night were combined, by taking the median of the available frames, and rebinned, in order to improve the signal-to-noise ratio and to provide manageable spatial resolutions to perform model analysis. Except for the NMSO image for which absolute calibration is not available, we converted the images to solar disk intensity units appropriate for the analysis in terms of dust tail models. The 0.8-m IAC80, 1.23-m of the Centro Astronómico Hispano-Alemán (CAHA), 0.1-m of NMSO, and 1.50-m of the Observatorio de Sierra Nevada (OSN) telescopes were used to get information on the tail brightness and morphology, while the higher spatial resolution of the 4.2-m WHT telescope images on the 27th December were in principle used to search for small-sized fragments possibly generated after the event. However, and owing to significant background in the images by moonlight, our limiting $V$ magnitude on the individual images was $V=20.5 \pm 0.3$. Assuming a fragment having the same $p_{v}=0.038$ as Scheila, and applying the formalism of Bowell et al. (1989) with a slope parameter of $G=0.076$ (Warner 2010), we get a minimum fragment diameter of $5.6 \pm 0.8 \mathrm{~km}$. No fragments of this size of larger were detected near the asteroid. On the other hand, the tail was not detected neither on the CAHA images of January 15th, nor on the OSN images of January 25th, 2011.

Figure 1 depicts combined images of (596) Scheila acquired at the 0.8-m IAC-80 telescope of the Observatorio del Teide (Izaña, Canary Islands), on 13th and 14th December 2010, at the 1.23-m telescope of CAHA, on December 17th 2010, and at NMSO on December 29th. The images, that are oriented in the $(N, M)$ system (Finson \& Probstein 1968), show a double tail in the sunward direction. The tails are not symmetric, the northern (left) one being broader and extending over more length than the southern (right) one. An antisunward, moderately bright, spike is also seen to emerge near the optocenter, which is clearly discernible in the first three dates of observation.

\section{The Model}

The asteroid is assumed to be a spherical body of radius $R_{n}=56.67 \mathrm{~km}$ with a density of $1500 \mathrm{~kg} \mathrm{~m}^{-3}$. This value is close to the density estimates of several D-type or P-type asteroids (P-type are similar to D-type asteroids in having low albedo and a featureless reddish electromagnetic spectrum). Thus, the most recent estimates for the martian satellites Phobos and Deimos have $1530 \pm 100 \mathrm{~kg} \mathrm{~m}^{-3}$ and $1340 \pm 830 \mathrm{~kg} \mathrm{~m}^{-3}$, respectively (Smith et al. 1995). These bodies are assumed to be captured D-type asteroids from the Main Asteroid Belt (MAB) (Hilton 2002). Asteroid (3415) Danby, which is classified as P- or D-type, has a 
density $\rho>1400 \mathrm{~kg} \mathrm{~m}^{-3}$ (Dahlgren et al. 1998). However, higher densities $\left(2500 \mathrm{~kg} \mathrm{~m}^{-3}\right)$ have been reported for D-type L4-trojan contact-binary (624) Hektor asteroid (Weidenschilling 1980; Lacerda \& Jewitt 2007), while a lower density (1050 $\pm 330 \mathrm{~kg} \mathrm{~m}^{-3}$ ) has been reported for the P-type binary L5-Trojan system (617) Patroclus-Menoetius (Mueller et al. 2010). For an asteroid having the size and density assumed, the gravitational force exerted on a particle in its neighborhood is not negligible compared to Sun's gravity at 3.1 AU. This implies that this force must be taken into account when computing the orbits of particles ejected from the asteroid surface. We simulate the short-duration event as a collection of particles being ejected from the surface at a given direction and velocity, being immersed in both the asteroid and solar gravity fields, and subjected to the solar radiation pressure. In a heliocentric inertial frame, with $x$-axis pointing to the asteroid perihelion, $z$-axis perpendicular to the orbit plane in the direction of the asteroid orbital angular momentum, and $y$-axis perpendicular to both $x$ - and $z$-axis forming a right-handed Cartesian coordinate system, the equation governing the motion of the particles can be written as:

$$
\frac{\mathrm{d}^{2} \mathbf{r}_{\mathbf{s}}}{\mathrm{d} t^{2}}=-G M_{a} \frac{\mathbf{r}_{\mathbf{a}}}{r_{a}^{3}}-\mu G M_{s} \frac{\mathbf{r}_{\mathbf{s}}}{r_{s}^{3}}
$$

where $G$ is the gravitational constant, $M_{a}$ is the asteroid mass, $M_{s}$ is the mass of the Sun, $\mathbf{r}_{\mathbf{s}}$ is the position vector of the particle, $\mathbf{r}_{\mathbf{a}}$ is the vector from the asteroid to the particle, and $t$ is time. The parameter $\beta=1-\mu$ is the ratio of the solar gravity force to the pressure radiation force and is given by $\beta=C_{p r} Q_{p r} /\left(2 \rho_{d} r_{d}\right)$, where $C_{p r}=1.19 \times 10^{-3} \mathrm{~kg} \mathrm{~m}^{-2}$, and $\rho_{d}$ is the particle density, assumed at $\rho_{d}=1000 \mathrm{~kg} \mathrm{~m}^{-3}$. For absorbing particles of radii $r_{d}>$ $0.25 \mu \mathrm{m}$, the radiation pressure coefficient is $Q_{p r} \sim 1$ (Burns et al. 1979).

The initial position and velocity of the particle on the asteroid surface are input parameters. The rotation period of the asteroid is estimated at $15.877 \pm 0.005 \mathrm{~h}$ (Warner 2011), so that the maximum linear velocity (i.e., at the equator) is $6.2 \mathrm{~m} \mathrm{~s}^{-1}$. However, for the asteroid size and density assumed, the escape velocity is of order $50 \mathrm{~m} \mathrm{~s}^{-1}$, so that the relative influence of asteroid rotation in modifying the ejection velocity amounts to $\sim 12 \%$ at most. The low rotation period also indicates that the asteroid cannot be undergoing mass loss from rotational instabilities.

For simplicity, and since the orientation of the rotation axis of the asteroid is unknown, we assumed that the asteroid is rotating with the spin axis perpendicular to the orbit plane. We also assumed that the event took place on a specific region of the asteroid surface, characterized by a latitude $\theta$ and longitude $\phi$, reckoned from the asteroid subsolar meridian at perihelion. Playing with the three parameters $\theta, \phi$, and outburst time, which is constrained to be between November 11 and December 3, we arrived to the conclusion that $\theta=-10^{\circ}$ and 
$\phi=40^{\circ}$ are good choices if the event time is set around November 27th, 2010. We will show later that changing this date significantly will result in a ejecta distribution incompatible with the observations. However, other solutions are surely possible as well. For instance, if the event time changes by a significant fraction of the rotational period, the event longitude must be set different. Since the exact event time is very hard to determine, the longitude of impact is also undetermined. We characterize the direction of the ejected particles by an ejection cone with vertex at coordinates $(\theta, \phi)$, and having an opening angle between $0^{\circ}$ and $90^{\circ}$ with respect to the radial direction. The integration of the equation was performed by a fourth-order Runge-Kutta method with a variable time step proportional to the distance of the particle to the asteroid. The position of the asteroid itself was computed by keplerian dynamics from its orbital elements. The trajectories of a large number of particles $\left(\sim 10^{5}\right)$ were computed, and their positions at the different observational times determined. Their positions in the heliocentric system were then converted to the asterocentric system $(\xi, \eta, \zeta)$ and finally converted to the photographic $(N, M)$ plane by computing the asterocentric coordinates of the Earth $\left(\xi_{e}, \eta_{e}, \zeta_{e}\right)$ at the observation time, using the equations by Finson $\&$ Probstein (1968). The cross section of a spherical dust grain, $C=\pi r_{d}^{2}$, expressed in $\mathrm{m}^{2}$, is related to its magnitude in the filter bandpass, $m$, by the following expression:

$$
p_{v} C=2.238 \times 10^{22} \pi R^{2} \Delta^{2} 10^{0.4\left(m_{0}-m\right)}
$$

where $p_{v}$ is the albedo times the phase function, $R$ is the asteroid heliocentric distance in Astronomical Units (AU), $\Delta$ is the asteroid geocentric distance in $\mathrm{AU}$, and $m_{0}$ is the magnitude of the Sun in the filter bandpass. We assumed $p_{v}=0.04$, i.e., the same value as for the bulk asteroid. For higher values of $p_{v}$, the derived ejected mass would be lower. The escaping mass is also dependent on the grain size distribution and its assumed minimum and maximum radius. For simplicity, we adopted a power-law size distribution, with a power index to be determined in the fitting procedure. We assumed a wide size distribution with $r_{d}(\min )$ in the sub-micrometer range and $r_{d}(\max )$ in the centimeter range.

As usual, the ejection velocity of the particles is assumed to follow a dependence on the $\beta$-value of the kind $v=v_{0} \beta^{\gamma}$, where $v_{0}$ and $\gamma$ must be determined, and subjected to the condition $v \geq v_{e s c}$, where $v_{e s c}$ is the escape velocity at the asteroid surface, $v_{e s c}=\sqrt{\frac{2 G M_{a}}{R_{n}}}$. We found that the best solutions are attained when we set a limit of the maximum velocity for the smallest particles. Specifically, for $\beta>0.1$, we set the particle velocity as equal to the value at $\beta=0.1$, i.e., $v=80 \mathrm{~m} \mathrm{~s}^{-1}$.

As stated above, the particle ejection is assumed to occur within an emission cone, where all directions are equally probable, in principle. Thus, each particle is emitted in a 
direction forming a random angle in the $0^{\circ}-90^{\circ}$ domain with the direction of local zenith at coordinates $(\theta, \phi)$.

\section{Results and discussion}

Since the computational time of the Monte Carlo process is long owing to the large number of ejected particles whose trajectory must be integrated to have a good statistics, we limited the search in the parameter space to a minimum. A list with all input parameters, their range searched, and their best estimates, is given in Table II. For each parameter, the range searched was set to have reasonable limiting values. We remark that alternative scenarios possibly exist, the resulting best parameter set of table II being just one that fits the data with relative accuracy.

The selection of best-fit parameters was made after a long trial-and-error experimentation with the input parameters. We first built synthetic images for a modest number (say, $10^{3}$ ) of particles ejected in the Monte Carlo process, in order to check the spatial extent of the synthetic images at each date in comparison with the observed images, their overall shape, and the resulting fluxes. The projection of the particles ejected on the $(N, M)$ photographic plane is shown in figure 2, for the best fit parameters. Figure 2 shows the location in the $(N, M)$ plane of a small number of particles ejected assuming the best-fit parameters shown in the last column of table II. In this figure, two contours of Scheila's image on December 13 th are also drawn for comparison. The emission cone is assumed to be located at $-10^{\circ}$ latitude, and we have plot separately the particles being emitted through different cone angle (denoted by $\Upsilon$ ) bins. Our purpose is to show the dependence of particle ejection cone angle on the projected final positions on the $(N, M)$ plane. Only some specific cone angle bins have been displayed for clarity. Particles ejected within cone angles near $\Upsilon=0^{\circ}$, i.e., towards the local zenith, are essentially aligned with the antisolar direction, while particles emitted near the tangent direction (ejected in the interval $\Upsilon=\left[80^{\circ}, 90^{\circ}\right]$ ) are defining the outer shell of the ejecta. The overall spatial extent of the ejected particles match approximately the dimensions of the outer contour of the observed image, and the same happens for all the other observing dates. Concerning the size distribution of the particles ejected, Jewitt et al. (2011) have proposed a much narrower distribution, with particles having maximum radii of 1-10 $\mu \mathrm{m}$. For such a narrow distribution, we could not fit neither the spatial extent of the observed images nor their brightness distribution, however. Figure 3 shows the location on the $(N, M)$ plane, for the December 13 image, of particles in the 0.1 to $10 \mu \mathrm{m}$ range ejected on November 27, 2010, and December 3, 2010 (the latest outburst date compatible with the observations by Larson (2010)), with ejection velocities randomly distributed between the 
escape velocity and twice that value. As it is seen, the distribution of the particles in the $(N, M)$ plane does not correlate with the observations, with regions in the observed image that are not populated with particles of that size range, implying a wider size distribution function, such as stated in table II. The situation does not improve at all for other possible ejection dates or higher ejection velocities than twice the escape velocity.

There are, however, some issues to be addressed before the proposed ejection scenario is considered as plausible. As stated above, in the observed images we detected two tail arms and a central "spike", that the tail model should reproduce. The central "spike" could be due to the emission cone near $\Upsilon=0^{\circ}$, while the arms could be associated to some ejection asymmetry within the cone. Since the asterocentric latitude of the Earth on December 13th is very small $\left(\sim 1^{\circ}\right)$, and based in the assumption that the asteroid equator is on the orbital plane, the arms must be associated to some kind of asymmetry in the direction of the $z$ axis in the asterocentric system. Thus, we have found that restricting the emission angles respect to the asterocentric $z$ axis to be less than $60^{\circ}$, if $\Upsilon<40^{\circ}$ or if $\Upsilon>60^{\circ}$, the pattern displayed by the ejected particles is morphologically very similar to the observed image (see figure 4). Interestingly, the range of ejection angles $45^{\circ}-60^{\circ}$ define a full $360^{\circ}$ cone that might corresponds to the ejection cone which is normally caused by an impact, while the asymmetry in the $z$ direction might corresponds to ejecta asymmetries caused by an oblique impact in the $+z$ sense. This is just tentative, as the information at hand is not complete enough as to permit deriving any firm conclusion. Also, this is just a first approximation of the problem: the three-dimensional nature of the ejection should be surely more complicated than is simply derived by restricting emission along a specific direction.

Another remarkable issue is the presence of the central spike. In our model, we have identified this spike with ejection near $0^{\circ}$, i.e., along the local vertical at the impact site. In our simulations, this spike is considerably brighter than observed. However, we did not account for the fact that part of the material in this direction, if an impact is assumed as the cause of the event, could be associated to spallation (Melosh 1984), and, if so, the ejection velocity of the spalled material would have been much higher than assumed and the brightness of the spike would be reduced accordingly. Again, this hypothesis is to be taken with caution.

The outburst date is constrained to occur on November 27th. Figure 5 shows the resulting positions on the $(N, M)$ plane relative to the observed images at different dates, assuming the outburst date on November 20th, 27th (our best fit), and December 2nd. Let us recall that the outburst date is limited by the observations to be between November 11, and December 3rd (Larson 2010). The assumption of an outburst time earlier than the best fit date implies an ejecta distributed in a larger area than observed, while if that date 
is assumed later, the ejecta will cover a smaller area, as expected. We estimate that the outburst date is November 27th, with an accuracy of \pm 3 days.

The particle ejection velocities we have found range from $\sim 50 \mathrm{~m} \mathrm{~s}^{-1}$ to $\sim 80 \mathrm{~m} \mathrm{~s}^{-1}$, for the particle size domain $5 \mathrm{~cm}$ to $8 \times 10^{-5} \mathrm{~cm}$, respectively. The power-law index of the ejection velocity versus $\beta$ (proportional to the inverse of particle radius) is very low, $\gamma \sim 0.05$ (see table II). In this context, it is important to consider that models of particle ejection by "regular" cometary ice sublimation (e.g. Fulle et al. 2010), or by "outburst" comets, such as 29P or 17P (Moreno et al. 2008; Moreno 2009), where hydrodynamic gas drag holds, result in power indexes of order 0.5 , so that the largest particles tend to move considerably slower in relation to the smallest particles than that found for this Scheila's event, where all particles move at much more comparable speeds among them.

From laboratory experiments, a correlation between mass and velocity of ejected fragments from a collision event in the form of a power law has been reported by some authors (e.g., Nakamura \& Fujiwara 1991; Giblin 1998). Although Nakamura \& Fujiwara (1991) obtained $v \propto m^{-1 / 6}$, Giblin (1998) reported that such correlation must be considerably weaker, with power-law exponents in the range $-1 / 6$ to 0 , with an average of $-1 / 13$. Nakamura et al. (1994) also reported that the velocity laws can be extrapolated for the measured $\mathrm{mm}$ to $\mathrm{cm}$ range to smaller size ranges. Also, it is important to consider the magnitude of the velocities found. Let us assume an impact with a body at the mean velocity in the MAB $\left(\sim 5 \mathrm{~km} \mathrm{~s}^{-1}\right.$, Farinella \& Davis 1992). Collision experiments at comparable speeds show that the maximum measured ratio of ejection velocities to impactor velocity is or order $v / U \sim 0.01$ (e.g. the experiments on dry sand by Cintala et al. 1999, or Housen \& Holsapple 2011), or larger (experiments on glass $\mu$-spheres, Yamamoto et al. 2005), so that we would get maximum velocities of $\mathrm{v} \approx 50 \mathrm{~m} \mathrm{~s}^{-1}$, i.e., in agreement with our estimates.

Based on the particle ejection velocity arguments posed in the previous paragraphs, we conclude that a collision event is a likely cause of the Scheila's outburst. If the impact took place at the mean velocity in the MAB of $\sim 5 \mathrm{~km} \mathrm{~s}^{-1}$, an estimate of the impactor mass can be obtained from scaling laws (Housen et al. 1983). In what follows, and for lack of additional information, we will assume that the projectile density is of the same order as the target. The ejected mass having velocity higher than a certain value $M(v)$ divided by the impactor mass $m_{i}$ can be related to the ratio $v / U$, depending on the target strength (Housen \& Holsapple 2011). Thus, experiments show that for $v / U \sim 0.01, M(v) / m_{i} \sim 4$ for weakly cemented basalt and $M(v) / m_{i} \sim 100$ for solid basalt (Housen \& Holsapple 2011). As the model predicts a total ejected mass of $2 \times 10^{10} \mathrm{~kg}$, we would get $m_{i}$ in the range $5 \times 10^{9}$ $\mathrm{kg}$ to $2 \times 10^{8} \mathrm{~kg}$. Converting these masses to sizes, with $\rho_{i}=1500 \mathrm{~kg} \mathrm{~m}^{-3}$, we get a spherical projectile in the range $\sim 30-90 \mathrm{~m}$ in radius. 


\section{Conclusions}

We have investigated whether a short-term duration event model for the outburst of (596) Scheila detected on December 2010 can match the observations. Integrating numerically the equation of motion of the ejected particles, we have found a set of physical parameters compatible with the observed morphology and brightness of the tail at different times after the event. We emphasize that this solution might not be unique, due to the large number of physical parameters involved. The total ejected mass turns out to be $2 \times 10^{10} \mathrm{~kg}$, the particles being distributed with a power-law of index -3 . The modeled ejection velocities giving rise to the observed tails $\left(\sim 50-80 \mathrm{~m} \mathrm{~s}^{-1}\right)$ and the weak power index relating ejection velocity with size $(\gamma=0.05)$ are compatible with a collision at the mean velocity in the MAB $\left(\sim 5 \mathrm{~km} \mathrm{~s}^{-1}\right)$. The ejecta is found to be composed of particles symmetrically distributed in an ejection cone of $45^{\circ}-60^{\circ}$ aperture, plus an asymmetric branch distributed along the axis perpendicular to the orbiting plane, probably associated to an oblique impact. The impact is estimated to occur on November 27th, with an estimated accuracy of \pm 3 days. By using scaling laws, and assuming the same density for the impactor as for the asteroid $(\rho=1500 \mathrm{~kg}$ $\mathrm{m}^{-3}$ ) the projectile size is estimated to lay in the $30 \mathrm{~m}$ to $90 \mathrm{~m}$ radius range.

This article is partially based on observations made with the IAC-80 operated on the island of Tenerife by the Instituto de Astrofísica de Canarias in the Spanish Observatorio del Teide; on the WHT operated on the island of La Palma by the Isaac Newton Group of Telescopes in the Spanish Observatorio del Roque de los Muchachos; on observations made with the 1.23-m telescope of the Centro Astronómico Hispano-Alemán at Calar Alto, operated jointly by the Max-Planck-Institut für Astronomie and the Instituto de Astrofísica de Andalucía (CSIC); on observations with the 1.5-m telescope of the Observatorio de Sierra Nevada, of the Instituto de Astrofísica de Andalucía (CSIC); and on observations at the New Mexico Skies Observatory, by courtesy of Joseph Brimacombe, who is gratefully acknowledged. We are also indebted to the OSN staff members, especially to F.J. Aceituno, V. Casanova, and A. Sota for carrying out the observations at the OSN.

This work was supported by contracts AYA2007-63670, AYA2008-01720E, AYA200908190, and FQM-4555 (Proyecto de Excelencia, Junta de Andalucía). J. Licandro, V. AlíLagoa, and O. Vaduvescu gratefully acknowledge support from the Spanish "Ministerio de Ciencia e Innovación" project AYA2008-06202-C03-02. J. L. Ortiz gratefully acknowledges support from the Spanish "Ministerio de Ciencia e Innovación" project AYA2008-06202-C0301. 


\section{REFERENCES}

Bodewits, D., Kelley, M.S., Li, J.Y., Landsman, W.B., Besse, S., \& A’Hearn, M. 2011, ApJ, 733, L3

Bowell, E., Hapke, B., Domingue, D., Lumme, K., Peltoniemi, J., \& Harris, A. W. 1989, Asteroids II (Tucson, AZ: Arizona Univ. Press), 524

Burns, J.A., Lamy, P.L., \& Soter, S. 1979, Icarus, 40, 1

Cintala, M.J., Berthoud, L., Hörz, F. 1999, Meteorit. Planet. Sci., 34, 605

Dahlgren, M., Lahulla, J.F., Lagerkvist, C.-I., Lagerros, J., Mottola, S., Erikson, A., GonanoBeurer, M., \& Di Martino, M. 1998, Icarus, 133, 247

Farinella, P., \& Davis, D.R. 1992, Icarus, 97, 111

Finson, M., \& Probstein, R. 1968, ApJ, 154, 327

Fulle, M., Colangeli, L., Agarwal, J., et al. 2010, Astron. Astrophys., 522, 63

Giblin, I. 1998, Planet. Space Sci., 46, 921

Hilton, J.L. 2002, in Asteroids III, Univ. of Arizona Press, Tucson.

Housen, K.R., Schmidt, R.M., Holsapple, K.A. 1983, J. Geophys. Res., 88, 2485

Housen, K.R., Holsapple, K.A. 2011, Icarus, 211, 856

Hsieh, H.H., \& Jewitt, D. 2006, Science, 312, 561

Jewitt, D., Weaver, H., Mutchler, M., Larson, S., Agarwal, J. 2011, ApJ, 733, L4

Lacerda, P., \& Jewitt, D. 2007, AJ, 133, 1393

Larson, S.M. 2010, IAU Circ. 9188, 1

Licandro, J. et al. 2011, in preparation

Melosh, H.J. 1984, Icarus, 59, 234

Monet, D.G., Levine, S.E., Canzian, B. et al. 2003, AJ, 125, 984

Moreno, F., Ortiz, J.L., Santos-Sanz, P., Morales, N., Vidal-Núñez, M.J., Lara, L. M., Gutiérrez, P.J. 2008, ApJ, 677, L63 
Moreno, F. 2009, ApJS, 183, 33

Mueller, M., Marchis, F., Emery, J.P., Harris, A.W., Mottola, S., Hestroffer, D., Berthier, J., \& di Martino, M. 2010, Icarus, 205, 505

Nakamura, A., Fujiwara, A. 1991, Icarus, 92, 132

Nakamura, A., Fujiwara, A., Kadono, T. 1994, Planet. Space Sci., 42, 1043

Smith, D.E., Lemoine, F.G., \& Zuber, M.T. 1995, Geophys. Res. Letter, 22, 2171

Tedesco, E.F., Noah, P.V., Noah, M., \& Price, S.D. 2002, AJ, 123, 1056

Warner, B.D. 2010, CBAT, 2590

Warner, B.D. 2011, Minor Planet Bulletin, 38, 96

Weidenschilling, S.J. 1980, Icarus, 44, 807

Yamamoto, S., Okabe, N., Kadono, T., Sugita, S., Matsui, T. 2005, Lunar Planet. Sci. XXXVI. Abstract 1600 


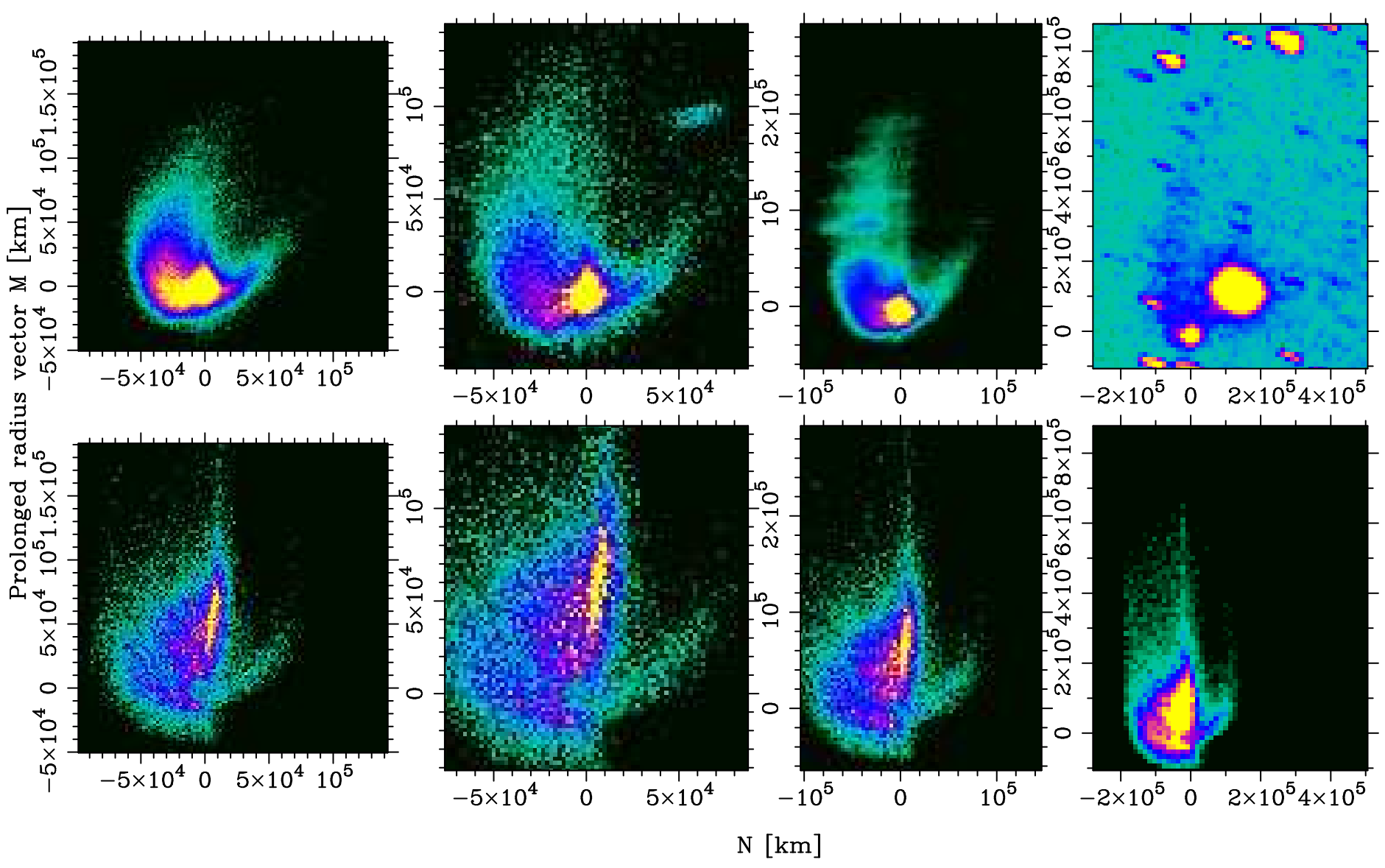

Fig. 1. - Left to right: Images of (596) Scheila corresponding to 13th, 14th, 17th, and 29th December, 2010 (see table II for exact times and telescopes used). The upper row corresponds to the observations, while the lower row to the models. The tails show clearly a bifid pattern with a central spike in the sunward direction, although it not detectable in the 29 December image. Except for this latter case, the modeled images are rendered using the same color code for the intensities as the corresponding observed images at the top row. 


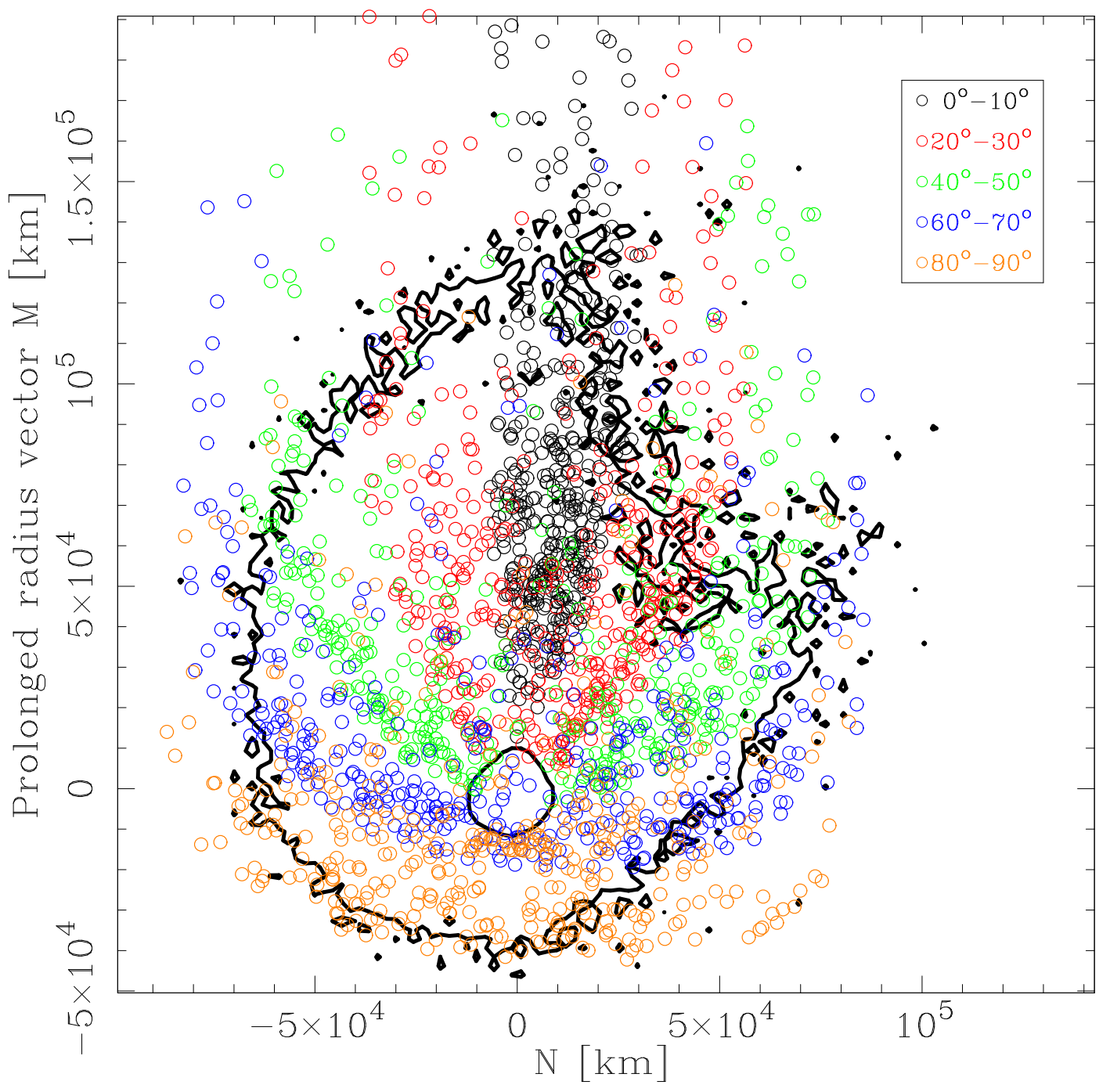

Fig. 2.- Black thick solid lines: contour plots at $10^{-14}$ and $7 \times 10^{-14}$ solar disk intensity units for Scheila's December 13th image. The open colored symbols denote the position on the $(N, M)$ plane of ejected particles from different cone angle bins as indicated, for the best fit model parameters (see table II). Only some specific cone angle bins have been drawn for clarity. 

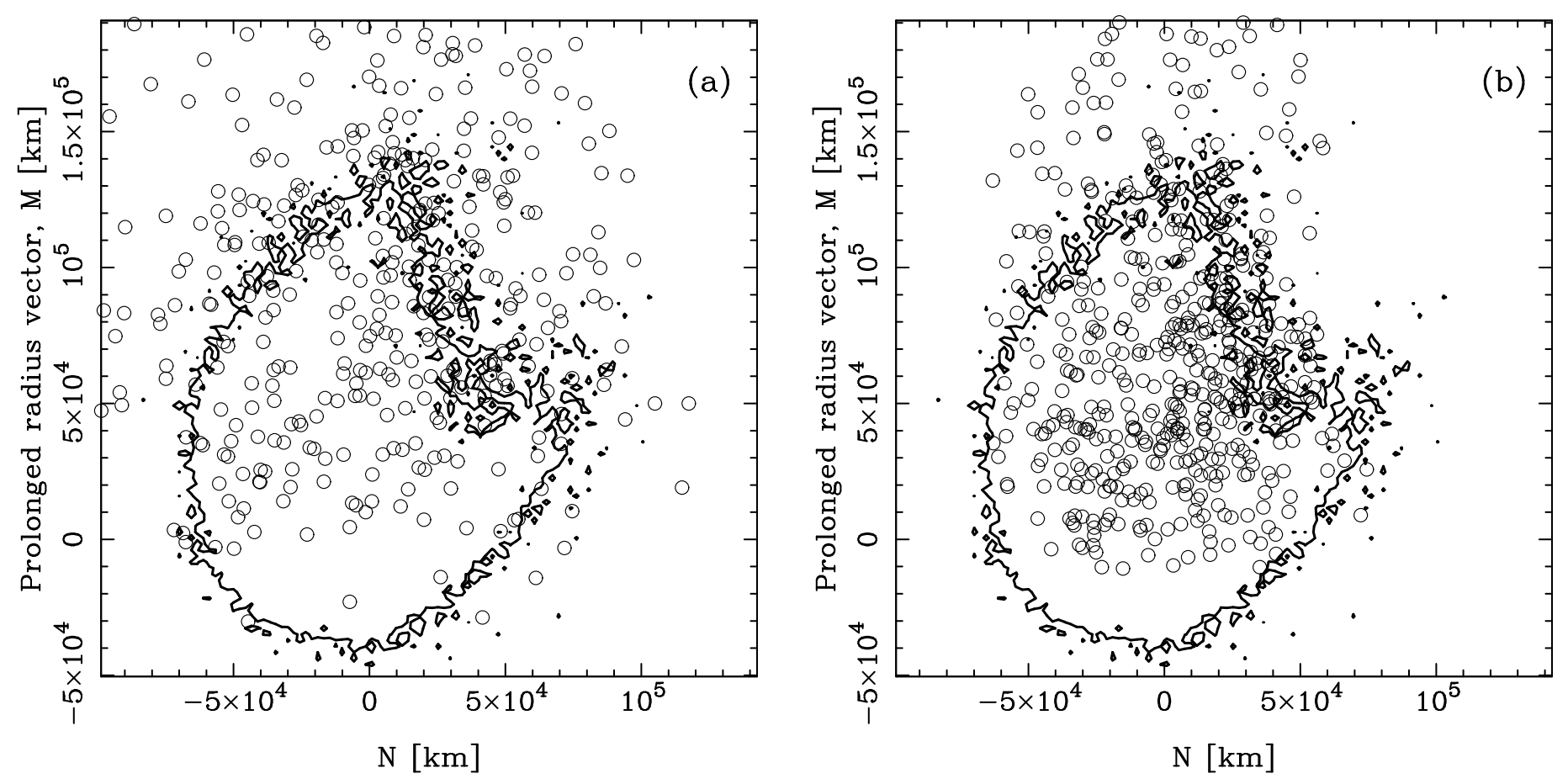

Fig. 3.- Black thick solid line: contour plots at $10^{-14}$ solar disk intensity units for Scheila's December 13th image. The open circles represent the position in the $(N, M)$ plane of 625 model particles ejected in November 27th, 2010 (a), and in December 3rd, 2010 (b). The particle ejection velocities are randomly distributed between the escape velocity and twice that value. 


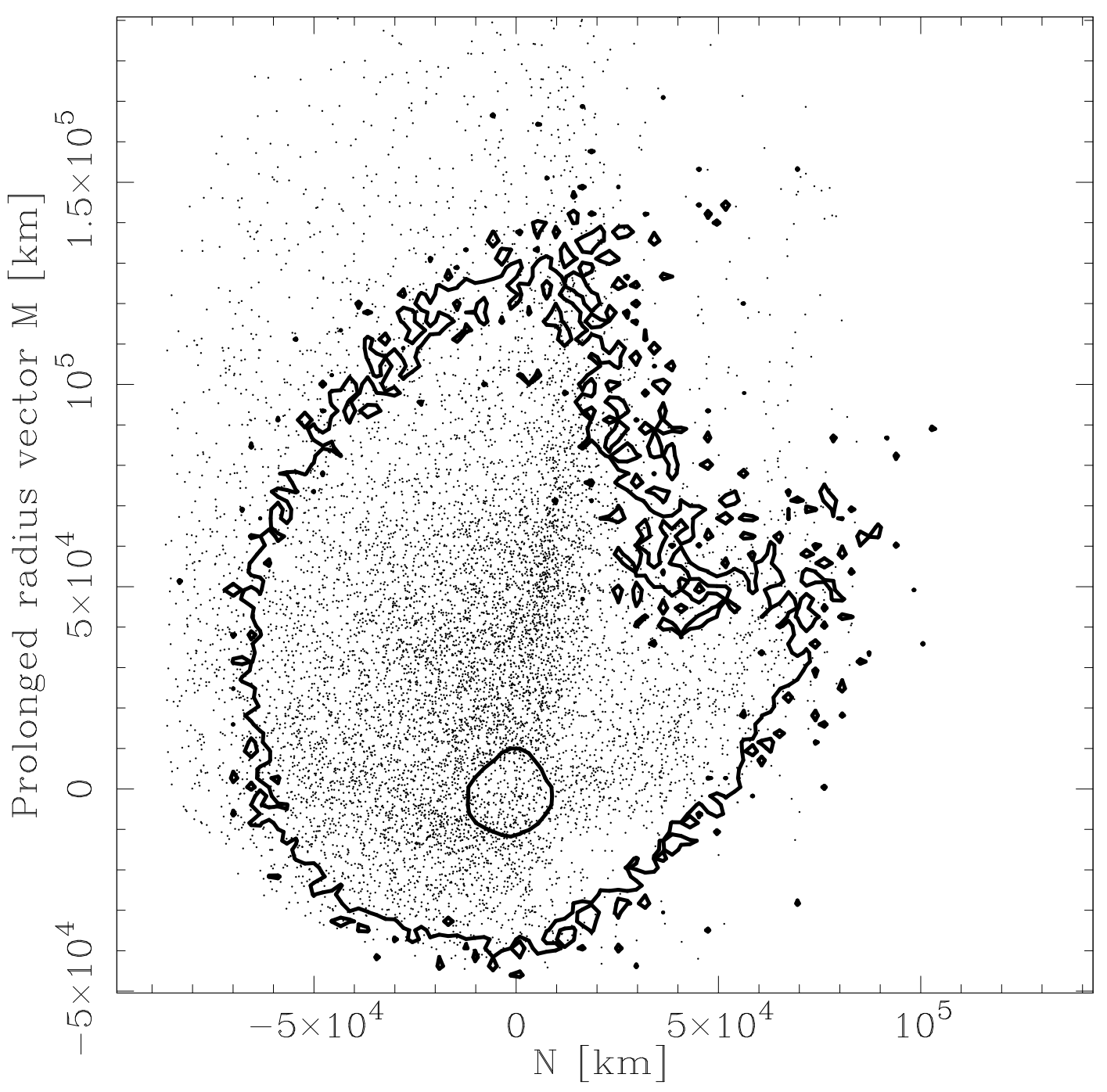

Fig. 4. - Black thick solid lines: contour plots at $10^{-14}$ and $7 \times 10^{-14}$ solar disk intensity units for Scheila's December 13th image. The dots represent the position on the $(N, M)$ plane of particles ejected for the best fit model parameters, but with some ejection restrictions along the $z$ asterocentric axis (see text). 

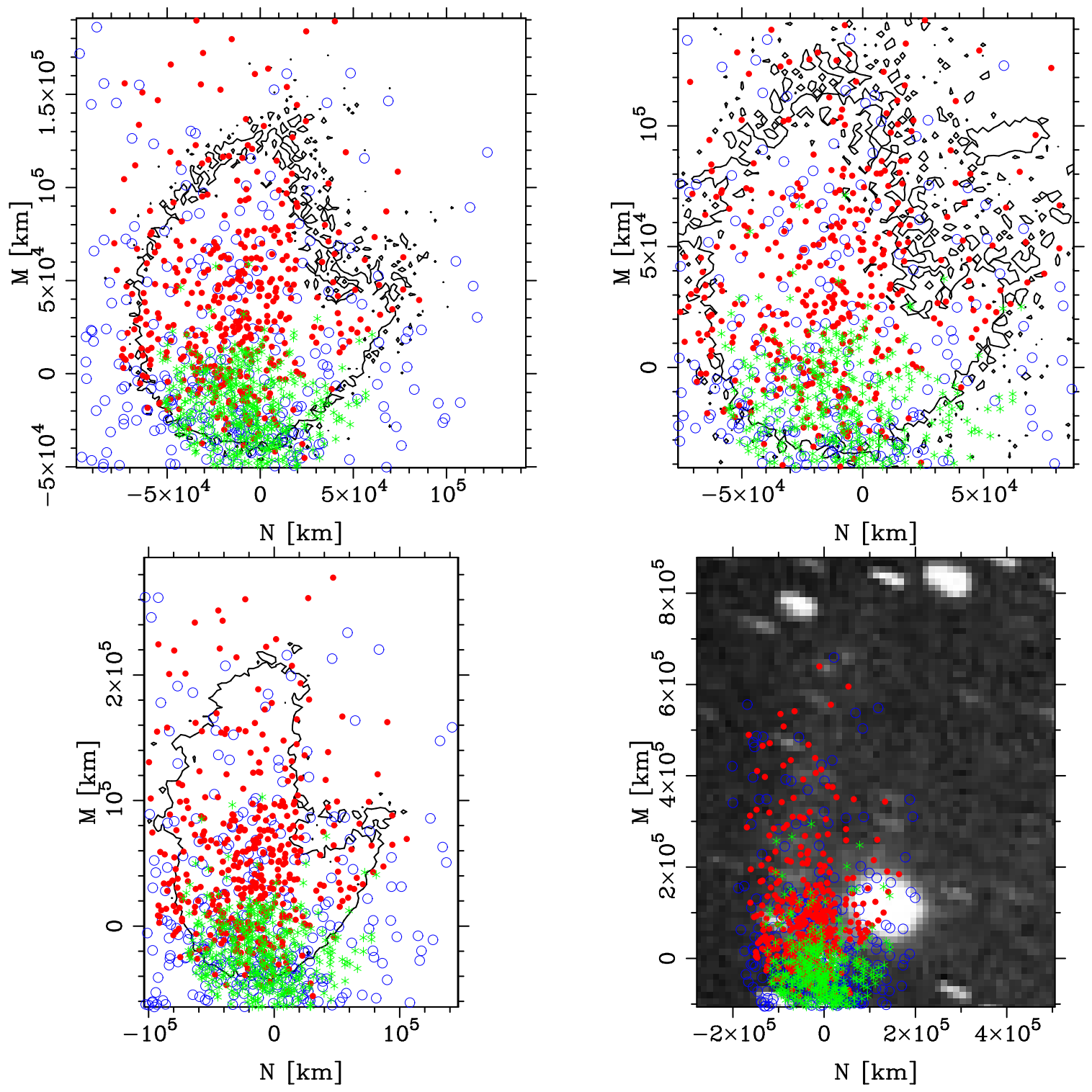

Fig. 5.- Blue, red, and green symbols correspond to the projections on the $(N, M)$ plane of particles ejected on November 20th, November 27th, and December 2nd, respectively, for the observations on December 13th (upper left panel), December 14th (upper right panel), December 17th (lower left panel), and December 29th (lower right panel). Contours of the observed images in are also drawn, corresponding to (solar disk units) $10^{-14}$ (upper left and upper right), and $5 \times 10^{-15}$ (lower left). The NMSU image (lower right) is not calibrated in intensity. 
Table 1. Observational circumstances of (596) Scheila. Position data include heliocentric distance $R$, geocentric distance $\Delta$, and phase angle $\alpha$. The pixel size refers to the spatial resolution of the final rebinned images used in the model analysis.

\begin{tabular}{cccccc}
\hline \hline UT date & Obs./Tel. aperture & $R(\mathrm{AU})$ & $\Delta(\mathrm{AU})$ & $\alpha^{\circ}$ & Pixel size $(\mathrm{km})$ \\
\hline 13.2 Dec 2010 & Teide IAC80 0.80 m & 3.106 & 2.521 & 16.27 & 2194 \\
14.2 Dec 2010 & Teide IAC80 0.80 m & 3.105 & 2.507 & 16.12 & 2182 \\
17.1 Dec 2010 & CAHA 1.23 m & 3.100 & 2.468 & 15.68 & 3580 \\
27.1 Dec 2010 & ORM WHT 4.2 m & 3.086 & 2.347 & 13.85 & - \\
29.3 Dec 2010 & NMSO FSQ-106ED 0.10m & 3.082 & 2.322 & 13.19 & 12980 \\
15.2 Jan 2011 & CAHA 1.23 m & 3.057 & 2.167 & 9.35 & - \\
25.1 Jan 2011 & OSN 1.5 m & 3.042 & 2.110 & 7.18 & - \\
\hline
\end{tabular}

Table 2. List of input parameters, their regions searched, and their best estimates.

\begin{tabular}{ccc}
\hline \hline Parameter & Domain searched & Best estimate \\
& & \\
\hline Impact date & Nov. 11 th to Dec. $3 \mathrm{rd}, 2010$ & Nov. $27 \mathrm{th}, 2010$ \\
\hline Minimum particle radius & $10^{-6}$ to $10^{-4} \mathrm{~cm}$ & $8 \times 10^{-5} \mathrm{~cm}$ \\
\hline Maximum particle radius & 1 to $10 \mathrm{~cm}$ & $5 \mathrm{~cm}$ \\
\hline Size dist. power index & -4 to -1.5 & -3.0 \\
\hline Total ejected mass & $10^{7}$ to $10^{11} \mathrm{~kg}$ & $2 \times 10^{10} \mathrm{~kg}$ \\
\hline Ejection velocity: & & $v_{0}=90 \mathrm{~m} \mathrm{~s}-1$ \\
$v(\beta)=v_{0} \beta^{\gamma}$ & $v_{e s c}$ to $100 \mathrm{~m} \mathrm{~s}-1$ & $-10^{\circ}$ \\
$v_{0}$ & 0 to 0.5 & $\gamma=0.05 ; v(\beta)=v(0.1)$ if $\beta>0.1$ \\
\hline Impact latitude: $\lambda$ & $-90^{\circ}$ to $90^{\circ}$ & \\
\hline
\end{tabular}

